\title{
Gastric hamartomatous inverted polyp mimicking ectopic pancreas on endoscopy and endosonography
}

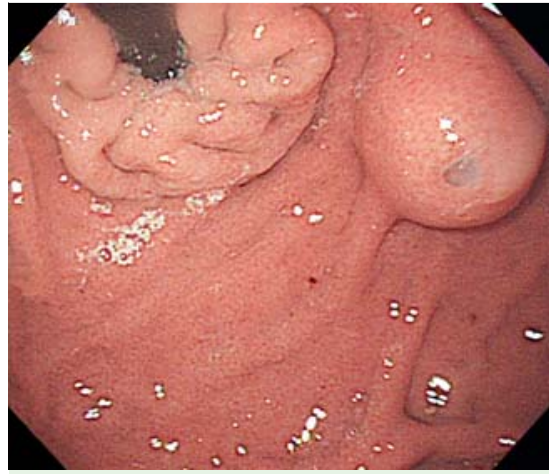

Fig. 1 Esophagogastroduodenoscopy disclosed an 18-mm submucosal tumor in the posterior wall of the gastric fundus, with whitish mucus flowing out from a central orifice.

Gastric hamartomatous inverted polyp (HIP) is difficult to diagnose preoperatively because of its rarity [1,2]. It is pathologically benign, but has been reported as a paracancerous lesion [3-5], and therefore its diagnosis and treatment are clinically important. We report a case of gastric HIP and focus on its special characteristics on endoscopy and endosonography.

An 18-mm submucosal tumor with whitish mucus flowing out from a central orifice was an incidental finding in the posterior wall of the gastric fundus in a 23-year-old woman ( $\bullet$ Fig. 1). Endoscopic ultrasonography $(20 \mathrm{MHz})$ revealed a heterogeneous tumor arising from the third layer of the gastric wall with a cystic portion inside ( $\mathbf{F i g . 2 a}$ ). A $3.2 \mathrm{~mm}$ anechoic duct communicated between the surface and the cystic portion ( $\mathbf{F i g} \cdot \mathbf{2 b}$ ). Injection-assisted endoscopic mucosal resection was performed. Pathological analysis revealed overlying gastric mucosa with submucosal proliferation of cystic glands and smooth muscle bundles ( Fig.3a). The submucosal components connected with the overlying mucosa through a mucus-filling drainage duct ( Fig.3b). Immunohistochemical staining with actin demonstrated smooth muscle proliferation within the polyp ( Fig.3c). This picture was compatible with the diagnosis of gastric HIP.

To our knowledge, this is the first report of a gastric HIP with mucus secretion from

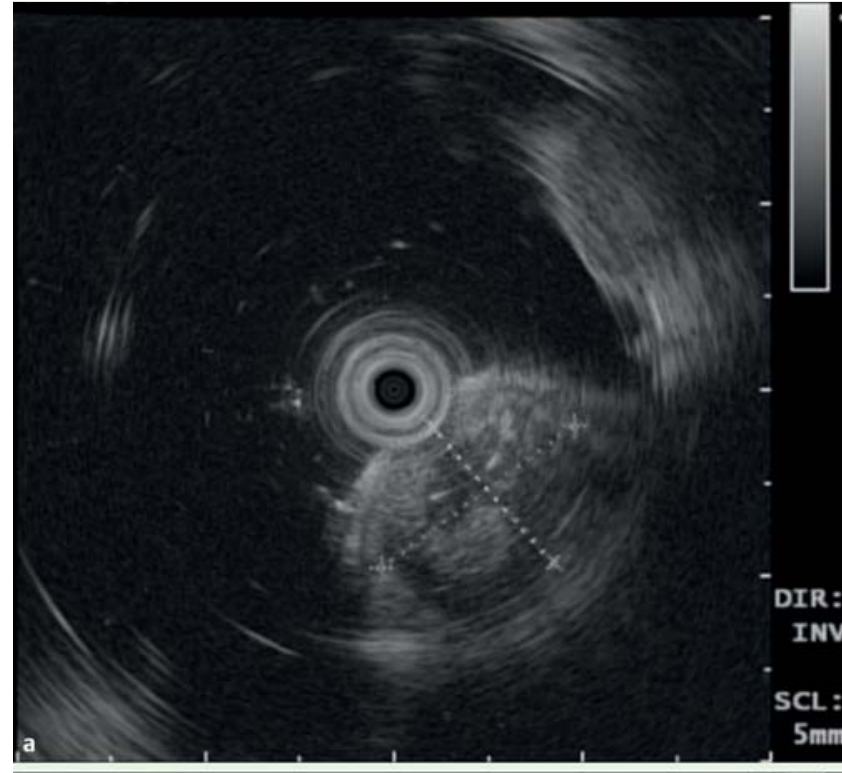

Fig. 2 a Endoscopic ultrasonography $(20 \mathrm{MHz})$ revealed a heterogeneous tumor arising from the third layer of the gastric wall, with a cystic portion inside. b A 3.2-mm anechoic duct (arrow) communicated between the surface and the cystic portion.

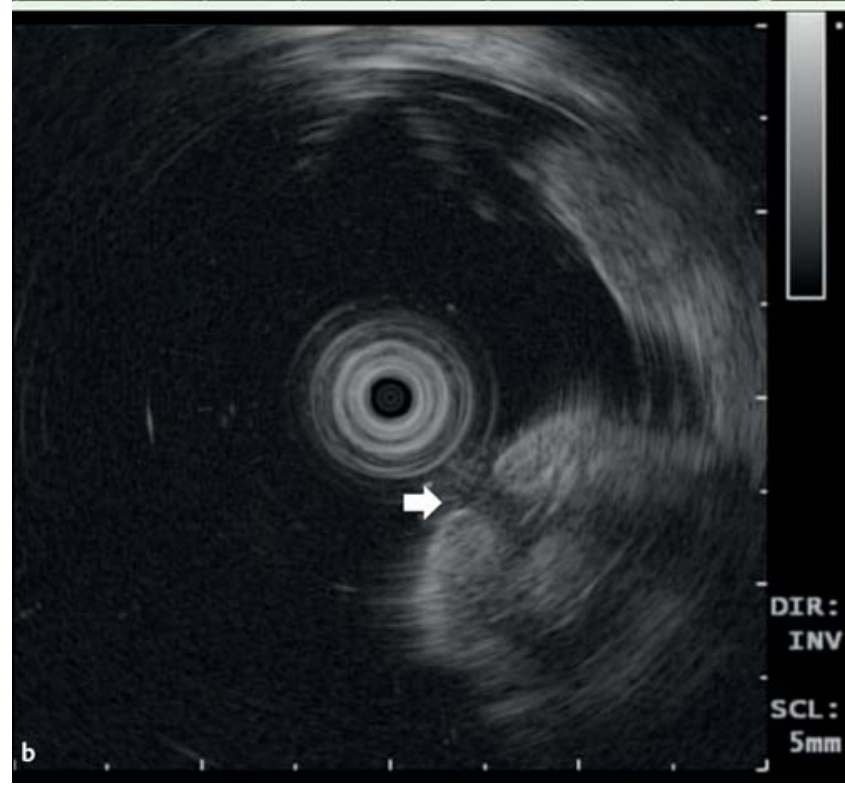

its central orifice on endoscopy and an anechoic duct on endosonography. Ectopic pancreases, carcinoid tumors, and hamartomatous polyps could manifest as central dimpling or umbilication on endoscopy. Occasionally, large gastrointestinal stromal tumors or leiomyomas also have a similar central depression or necrosis, due to insufficient blood supply [6]. However, mucus secretion and ductal structure on the image, corresponding to a drainage duct, are generally only seen in ectopic pancreases [7]. More than $90 \%$ of gastric ectopic pancreases are located in the antrum, which is different from our case [8]. Gastric HIP should therefore be considered as a possible diagnosis in cases of submucosal tumors located in the upper stomach, which have the potential to mimic ectopic pancreases.

Endoscopy_UCTN_Code_CCL_1AB_2AD_3AB

Competing interests: None 

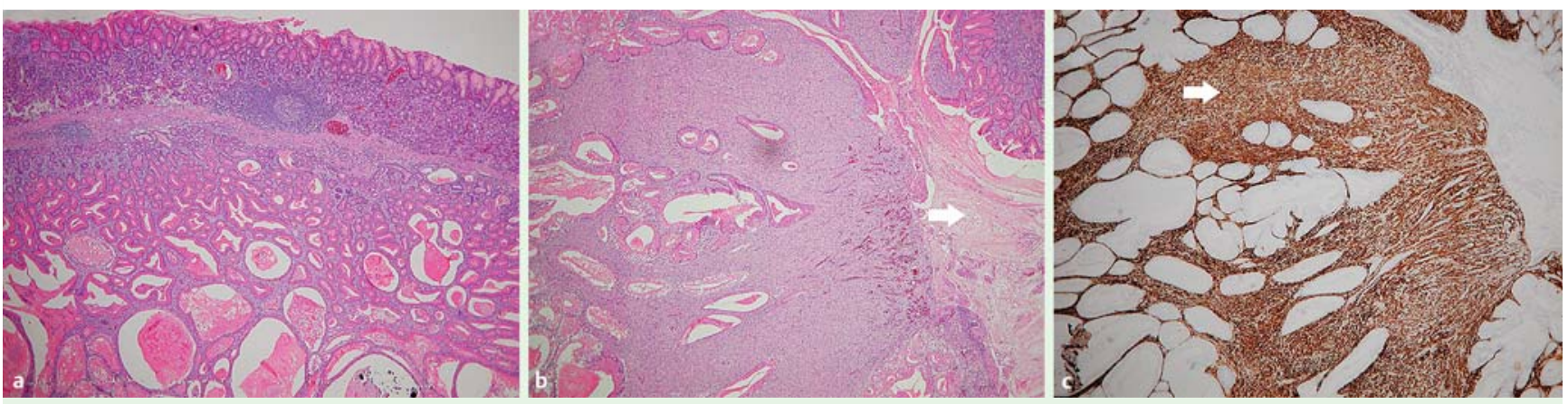

Fig.3 a Pathological analysis revealed overlying gastric mucosa with submucosal proliferation of cystic glands and smooth muscle bundles (H\&E stain, $\times 40$ ). b The submucosal components connected with the overlying mucosa through a mucus-filling drainage duct (arrow) (H\&E stain, $\times 100$ ). c Immunohistochemical staining demonstrated smooth muscle proliferation (arrow) within the polyp (actin stain, $\times 100$ ).

\section{Tsung-Chieh Yang', Ming-Chih Hou' ${ }^{1,2,3}$ Ping-Hsien Chen ${ }^{2,3}$, Wei-Chih Liao ${ }^{3,4}$, Anna Fen-Yau Li $\mathbf{i}^{3,5}$}

${ }^{1}$ Division of Gastroenterology, Department of Medicine, Taipei Veterans General Hospital, Taipei, Taiwan

${ }^{2}$ Endoscopy Center for Diagnosis and Treatment, Taipei Veterans General Hospital, Taipei, Taiwan

${ }^{3}$ School of Medicine, National Yang-Ming University, Taipei, Taiwan

${ }^{4}$ Division of Gastroenterology, Department of Medicine, Taipei Municipal Gan-Dau Hospital, Taipei, Taiwan

${ }^{5}$ Department of Pathology, Taipei Veterans General Hospital, Taipei, Taiwan

\section{References}

1 Yamashita M, Hirokawa M, Nakasono $M$ et al. Gastric inverted hyperplastic polyp. Report of four cases and relation to gastritis cystica profunda. APMIS 2002; 110: 717 723

2 Odashima M, Otaka M, Nanjo H et al. Hamartomatous inverted polyp successfully treated by endoscopic submucosal dissection. Intern Med 2008; 47: 259-262

$3 \mathrm{Oh} \mathrm{SJ}$, Oh CA, Kim DH et al. Adenocarcinoma derived from gastric hamartomatous polyps. J Korean Surg Soc 2011; 81: 419422

4 Ono S, Kamoshida T, Hiroshima Yet al. A case of early gastric cancer accompanied by a hamartomatous inverted polyp and successfully managed with endoscopic submucosal dissection. Endoscopy 2007; 39 (Suppl. 01): E202

5 Lin JC, Huang TY, Shih YL et al. Management of symptomatic hamartomatous polyps in stomach: analysis of a single center experience. J Med Sci 2013; 33: 29-36 Available at: http://jms.ndmctsgh.edu.tw/fdarticlee\% 5C3301029.pdf, accessed 2 January 2014

6 Classen M, Tytgat GNJ, Lightdale CJ. Gastroenterological endoscopy. 2nd ed. Stuttgart and New York: Thieme; 2010
7 Hawes RH, Fockens P. Endosonography. 2nd ed. Philadelphia, PA: Elsevier Saunders; 2011

8 Yie M, Jang KM, Kim MJ et al. Synchronous ectopic pancreases in the cardia and antrum of the stomach: a case report. J Korean Soc Radiol 2010; 63: 161 - 165

\section{Bibliography}

DOI http://dx.doi.org/

10.1055/s-0034-1364888

Endoscopy 2014; 46: E119-E120

(c) Georg Thieme Verlag KG

Stuttgart · New York

ISSN 0013-726X

\section{Corresponding author}

\section{Ming-Chih Hou, MD}

Endoscopy Center for Diagnosis and Treatment Taipei Veterans General Hospital

\#201, Section 2, Shipai Rd.

Beitou District

Taipei 11217

Taiwan

Fax: +886-2-77351329

mchou@vghtpe.gov.tw 